\title{
Automated Dust Devil Detection on Mars
}

\author{
By Kazunori OGOHARA, ${ }^{1)}$ Daiki Yasunaga, ${ }^{1)}$ and Genya Ishigami ${ }^{2}$ \\ ${ }^{1)}$ School of Engineering, University of Shiga Prefecture, Hikone, Japan \\ ${ }^{2)}$ Department of Mechanical Engineering, Keio University, Yokohama, Japan
}

(Received July 31st, 2015)

\begin{abstract}
A system for automated dust devil detection on Mars is developed based on images taken by the navigation camera aboard the Mars Exploration Rover, Spirit. This system enables us to pick up dust devils from the large number of images without checking each image visually and affords us a powerful tool for searching for dust devils and revealing their statistical properties for scientific studies. From an engineering point of view, the volume of dust devil image data to be downlinked to Earth for dust devil studies can also be drastically reduced by automated detection of dust devils because we can trim dust devil regions on the images onboard. A simple performance test using images taken on a particular sol shows that this system can detect almost all of the dust devils though also includes some false positives. Precision and recall are 0.85 and 0.81 , respectively, which are comparable with or more effective than those in previous studies.
\end{abstract}

Key Words: Mars, Dust Devil, Automated Detection

\section{Introduction}

Dust devils on Mars play a major part in maintaining the low (though higher than on Earth) background dust level in the atmosphere. ${ }^{1)}$ In particular, the atmospheric dust in the clear season (the northern summer) is considered to be primarily supplied by dust devils. Therefore, an understanding of the seasonality and regionality of dust devil activity and an estimation of the total amount of dust released by dust devils into the atmosphere are very important for studies of Martian meteorology and climatology.

Many dust devils have been observed by the previous landers, rovers, and orbiters on Mars. Mars Pathfinder observed many dust devils around the landing site, and the number, direction, estimated size, and opacity of dust devils have been summarized by Ref. 2). A navigation camera aboard the Mars Exploration Rover (MER), Spirit also imaged many dust devils over three Martian years. Refs. 3) and 4) report the size, local time, and traversal speed distributions of dust devils and the interannual and seasonal variations of dust devil occurrence. In contrast, few dust devils were observed by MER, Opportunity. The Mars Science Laboratory (MSL) also detected few visible dust devils. However, the Rover Environmental Monitoring Station (REMS) and the Mastcam aboard MSL have revealed that many invisible convective vortices occur at the landing site; these are dust devils that cannot lift dust from the surface. ${ }^{5}$ )

These observations and the theory of dust devil dynamics developed for terrestrial dust devils suggest that the number, size, lifetime, optical thickness, and frequency of dust devils depend heavily on atmospheric and surface conditions (temperature, surface albedo, thermal inertia, wind speed, surface roughness, atmospheric stability, season, latitude, etc.). In the case of Mars, the first step in quantitatively understanding dust supply by dust devils is to discover the correlation between dust devil activity and the atmospheric and surface conditions. Therefore, we need to extract dust devils from images obtained by the landers and rovers and investigate the atmospheric and surface conditions required for the observed dust devil activity. However, it is timeconsuming to extract dust devils from a vast number of images obtained at the Mars surface by checking the images visually, and thus we need a system by which we can obtain information on dust devils automatically.

At the same time, automation of dust devil detection has an important engineering benefit. The automation of dust devil detection and extraction allows us to drastically reduce the volume of data to be downlinked to the earth by clipping and sending detected dust devil areas. Refs. 6) and 7) describe the development of software that automatically extracts the scientifically important feature from among many candidates based on various criteria the scientist has specified in advance on the ground, thereby saving operation time and ensuring scientific results. Ref. 6) describes the development of "AEGIS," which extracts scientifically important rocks from a single image by automatically measuring the size, shape, and textures and pointing the instrument at the rocks. Ref. 7) developed an algorithm that automatically detects dust devils and clouds in several images obtained sequentially. However, their algorithm clearly overestimates the number of dust devils, and furthermore, they had difficulty evaluating the algorithm.

In this paper, we develop an algorithm for automatically detecting dust devils and storing information about their properties (the number, position, optical thickness, etc.). We focus on the extraction of dust devils from the many images already obtained by the previous rover for scientic purposes. In section 2 , we describe the image data we use in this paper and preprocess for extraction of dust devils. In section 3, a detailed procedure for dust devil detection is described. In section 4 , the algorithm developed in this paper is tested using Spirit/Navcam images and is shown to be effective in terms of some evaluation indices.

\section{Data}

We used 21 images (visible) acquired by the navigation cameras aboard the Spirit rover on Sol 0568 for this study (Table 1). All image data were ob- 
Trans. JSASS Aerospace Tech. Japan Vol. 14, No. ists30 (2016)

Table 1. List of images used in this study. These images were acquired by the Spirit/Navcam on Sol 0568 with a red filter, $580720 \mathrm{~nm}^{8}{ }^{8}$

\begin{tabular}{|c|c|c|}
\hline No. & Image file name & Observation start time \\
\hline 1 & 2n176788506radadaep156010c1.img & 2005-08-08T15:50:29.692 \\
\hline 2 & 2n176788526radadaep156010c1.img & 2005-08-08T15:50:50.192 \\
\hline 3 & 2n176788557radadaep156010c1.img & 2005-08-08T15:51:21.067 \\
\hline 4 & 2n176788589radadaep156010c1.img & 2005-08-08T15:51:53.192 \\
\hline 5 & 2n176788619radadaep156010c1.img & 2005-08-08T15:52:23.318 \\
\hline 6 & 2n176788653radadaep156010c1.img & 2005-08-08T15:52:57.443 \\
\hline 7 & 2n176788696radadaep156010c1.img & 2005-08-08T15:53:40.318 \\
\hline 8 & 2n176788730radadaep156010c1.img & 2005-08-08T15:54:14.193 \\
\hline 9 & 2n176788765radadaep156010c1.img & 2005-08-08T15:54:49.318 \\
\hline 10 & 2n176788799radadaep156010c1.img & 2005-08-08T15:55:23.568 \\
\hline 11 & 2n176788835radadaep156010c1.img & 2005-08-08T15:55:58.819 \\
\hline 12 & 2n176788869radadaep156010c1.img & 2005-08-08T15:56:33.069 \\
\hline 13 & 2n176788907radadaep156010c1.img & 2005-08-08T15:57:11.319 \\
\hline 14 & 2n176788950radadaep156010c1.img & 2005-08-08T15:57:53.694 \\
\hline 15 & 2n176788990radadaep156010c1.img & 2005-08-08T15:58:34.319 \\
\hline 16 & 2n176789033radadaep156010c1.img & 2005-08-08T15:59:17.445 \\
\hline 17 & 2n176789075radadaep156010c1.img & 2005-08-08T15:59:58.945 \\
\hline 18 & 2n176789118radadaep156010c1.img & 2005-08-08T16:00:42.195 \\
\hline 19 & 2n176789159radadaep156010c1.img & 2005-08-08T16:01:23.070 \\
\hline 20 & 2n176789199radadaep156010c1.img & 2005-08-08T16:02:03.571 \\
\hline 21 & 2n176789243radadaep156010c1.img & 2005-08-08T16:02:47.321 \\
\hline
\end{tabular}

tained through the Planetary Data System (PDS) website, http://pds-geosciences. wustl.edu/mer/ mer2-m-navcam-3-radiometric-sci-v1/mer2nc_1xxx/. The details of the engineering cameras aboard MER are summarized by Ref. citenbib08, and the calibration technique for the navigation camera instruments is described by Ref.9. Although the images are stored in 16-bit integer format, we converted them to floating-point format using RadianceScalingFactor and Radiance0ffset, included in each image's header. This enabled us to use images with a realistic radiance unit $\left(\mathrm{W} / \mathrm{m}^{2} / \mathrm{nm} / \mathrm{sr}\right)$.

\section{Automated Dust Devil Detection}

\subsection{Overview}

A system for automated dust devil extraction can enable scientists to automatically detect Martian dust devils on images without visually checking the images. When they begin their studies of Martian dust devils, researchers need to pick up dust devils individually from all images that have been obtained by the previous landers and rovers. Although a large number of dust devils have been observed around some landing sites, scientists must also look for dust devils in a much larger number of images. Such a manual search for dust devils is unrealistically timeconsuming. Our system enables us to extract dust devils without checking any images directly.

The system developed here performs four steps to detect dust devils. These steps are shown in Fig. 1 and are briefly described as follows.

- Subtraction from the background image. It is better to extract a dust devil from a difference image derived from an input image and another image obtained before the input image because a dust devil is usually obscured.

- Reversal of the sky in the difference image. In a difference image, the upper part of a dust devil above the horizon is scarcely distinguishable from the bottom part of another dust devil below the horizon as described in subsection 3.3 below. Therefore, we need to reverse the contrast of the sky in the difference image.

- Binarization. We need to binarize the difference image for dust devil detection. Note that it is difficult to automatically determine a suitable threshold value for brightness difference for the binarization.

- Post-proccessing. It is expected that the process described above will produce many false positives (e.g., shadows and tracks of dust devils that are included in the background image); we remove such false dust devils based on the aspect ratio and locations of detected dust devil regions.

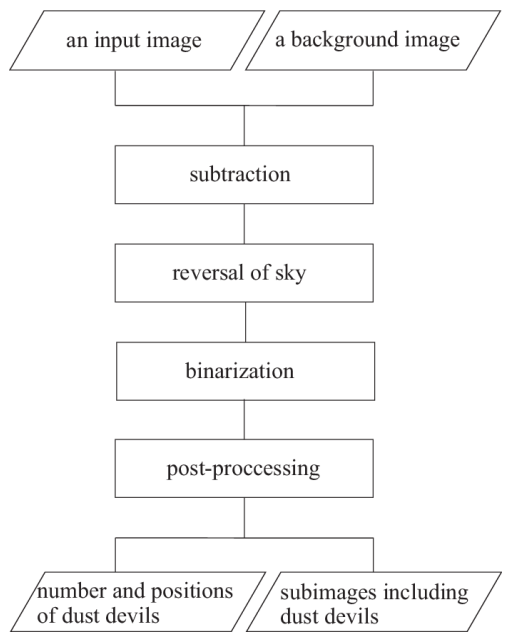

Fig. 1. Flow chart of the automated dust devil detection system developed in this study. 


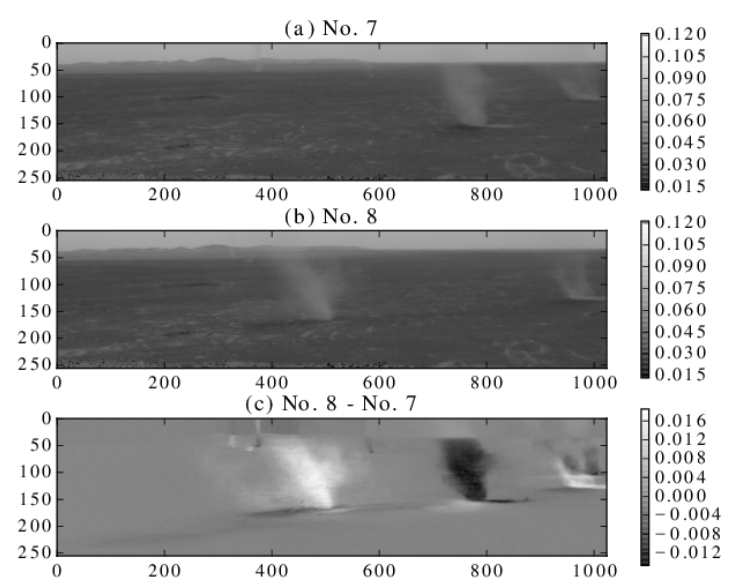

Fig. 2. Examples of (a) a background image (No. 7 in Table 1), (b) an input image (No. 8), and (c) a difference image.

\subsection{Subtraction from the background image}

A dust devil can be optically thin and more obscure than rocks and surface patterns around it. Therefore, it is very difficult to distinguish dust devils from other surface features in a single image. Dust devils usually pass laterally in the camera's field of view. The surface materials, on the other hand, are approximately invariant temporally. Because of the difference between dust devils and the surface in the time scale of changes, an effective way to extract a dust devil is to use a difference image derived from an input image and another image (hereafter called the background image) obtained before the input image. In this study, we use an image taken just before the input image as the background image. Figure 2 gives an example, showing the difference image obtained when the input image is No. 8 from Table 1 and the background image is No. 7.

\subsection{Reversal of the sky in the difference image}

A dust devil is usually brighter than the ground surface and darker than the sky. Therefore, the upper part of dust devils above the horizon on the input image and those on the background image appear as negative and positive signals on the difference image, respectively (Fig. 2(c)). In contrast, the bottom part of dust devils below the horizon on the input image and those on the background image appear as positive and negative signals on the difference image, respectively. This results in the false recognition of the upper part of a dust devil on the background image as a dust devil on the input image. In addition, the sky and the surface differ greatly in their brightness difference values in cases where the time interval between the two images is long. The sky may be indistinguishable from dust devils if we try to recognize dust devils based solely on brightness difference values. We therefore reverse the contrast of the sky in the difference image in order to display both parts of a dust devil on the sky and on the surface with the same sign.

In order to reverse the contrast of the sky in the difference image, we must first determine the horizon pixels. There is a large contrast in the $y$ direction between the sky and the ground surface including mountains. We obtained an edge image from the average image among the 21 images shown in Table 1 by applying a Prewitt filter in the $y$ direction and then regarding the pixel having the maximum edge value of each column as
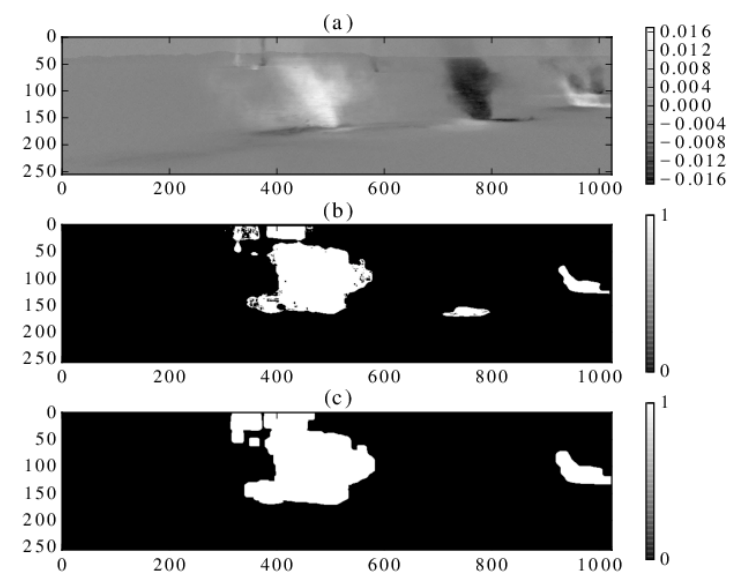

Fig. 3. Examples of (a) a reversed difference image obtained by subtracting image No. 7 from image No. 8, (b) the binarized image produced from (a), and (c) the final result of dust devil detection after the post-processing described in subsection 3.5 .

the horizon pixel for that column. If the horizon pixel of a column is five pixels or more from that in the column to the left, the horizon pixel of the column is regarded as being located in the same row as that of the column to the left. Next, the mode value of the brightness difference for the surface region is subtracted from that of the surface pixels in the difference image. Since most of the surface pixels correspond to clear areas rather than dust devils, dust devil features on the surface in the input and background images are displayed as positive and negative signals, respectively, in the image resulting from these processes. Then, the mode value of the brightness difference for the sky region is also subtracted from that of the sky pixels in the difference image; dust devil features on the sky in the input and background images are thus displayed as negative and positive signals, respectively. Finally, the sky pixels above the horizon pixels are multiplied by -1 . Figure 3 (a) shows the reversed difference image produced from Fig. 2(c).

\subsection{Binarization}

Dust devils on the reversed difference image described in the previous subsection are usually tiny features. Therefore, many methods for automated binarization (e.g., Otsu's method) do not work well. Some type of filter that can extract morphological features is required for dust devil detection using the reversed difference image. However, since a vast number of noisy false positives is expected, simple morphological operations (e.g., erosion and dilation) are not appropriate. A dust devil in a reversed difference image should appear to be a cluster of bright pixels, and positive noise a few pixels in size will probably tend to be distributed homogeneously on the image. Therefore, if more than $\alpha \%$ of pixels included in a small rectangular region whose center is at a current pixel is positive, the current pixel is set to 1 . The size of the small rectangular region is $9 \times 9$ pixels, and the value of $\alpha$ is 70 .

\subsection{Post-processing: removing false positives and count- ing dust devils}

We may find that many tiny foreground pixels still remain around relatively large positive areas that correspond to dust devils, as in Fig. 3(b). If we use this binarized image for label- 
ing and counting dust devils, we will find, erroneously, that a large number of dust devils occur and that most of the detected dust devils are extremely small. Therefore, after the binarization, we remove positive pixel areas having the width of a few pixels by using a $5 \times 5$ median filter. Further, the remaining positive pixels are removed or merged with each other by dilation and erosion. Finally, we label the dust devils sequentially. We first scan the binarized image pixel by pixel. For each foreground pixel:

We can find that many tiny fourground pixels still remain around relatively large positive areas which correspond to dust devils in Fig. 3(b). If we use this binarized image for labeling and counting dust devils, we falsely recognize that the large number of dust devils occur and most of the detected dust devils are extremely small. Therefore, after the binarization, we remove positive pixels with the width of a few pixels by $5 \times 5$ median filter. Furthermore, the remaining positive pixels are removed or merged with each other by dilation and erosion. Finally, we label dust devils sequentially. We first scan the binarized image pixel to pixel. If the pixel is foreground,

1. If only one of its eight neighbors has a label, this label is copied to the current pixel.

2. If all of its neighbors have the same label, this label is copied.

3. If the neighbors have different labels, one label is copied, and these labels are marked as equivalent.

4. If there are no labeled neighbors, a new label is assigned to the current pixel.

We scan the labeled image and replace all equivalent labels with a common label. However, tracks and shadows of dust devils in the background image still remain as foreground regions in the binarized image. These features are usually elongated and thus have a small aspect ratio. The final step in the present algorithm is to remove those elongated features that have an aspect ratio less than 0.4. In addition, dust devils a few pixels in size in the lower part of an image, which is the area close to the camera, are unrealistically small in size (m). Therefore, any detected dust devil regions 20 pixels in width or height in the lowest third of the image are filled with 0s. Figure 3(c) displays the final binarized image obtained by the procedure described in this section.

\section{Results and Discussion}

Table 1 shows a list of the Spirit/Navcam images used for this performance test and the observation start time for each. In Table 2, the number of dust devils detected using the method described in section 3 is listed as well as the number of dust devils the second author detected visually. Counts of true positives (TP), false positives (FP), and false negatives (FN) were 35, 6, and 8 , respectively, as shown in Table 2. TP here is the number of dust devils that can be recognized visually and detected automatically. FP is the number of those that are unrecognized visually but detected automatically, and FN is the number of those that can be recognized visually but are not detected automatically. Precision $p$, recall $r$, and F-measure $F$ were calculated as
Table 2. Results of automated dust devil extraction from the images listed in Table 1 . The numbers of dust devils detected visually and automatically are shown in the second and third columns, respectively.

\begin{tabular}{rrr}
\hline No. & Visual & Automated \\
\hline \hline 1 & 2 & 2 \\
2 & 3 & 3 \\
3 & 3 & 3 \\
4 & 2 & 2 \\
5 & 2 & 2 \\
6 & 4 & 4 \\
7 & 5 & 6 \\
8 & 4 & 4 \\
9 & 3 & 4 \\
10 & 4 & 1 \\
11 & 3 & 1 \\
12 & 3 & 3 \\
13 & 2 & 2 \\
14 & 1 & 1 \\
15 & 1 & 1 \\
16 & 1 & 1 \\
17 & 1 & 1 \\
18 & 0 & 0 \\
19 & 0 & 0 \\
20 & 0 & \\
21 & 0 &
\end{tabular}

follows:

$$
\begin{aligned}
p & =\frac{\mathrm{TP}}{\mathrm{TP}+\mathrm{FP}} \\
r & =\frac{\mathrm{TP}}{\mathrm{TP}+\mathrm{FN}} \\
F & =\frac{2 p r}{p+r}
\end{aligned}
$$

and are $0.85,0.81$, and 0.83 , respectively. F-measure is the harmonic mean between precision and recall. Usually, algorithms with high precision give low recall and vice versa. We can't evaluate the performance of the algorithms correctly if we adopt either of precision and recall as an evaluation index. Therefore, the harmonic mean between precision and recall, $F$, is often used for the evaluation of detection algorithms. $F$ remains relatively low if either precision or recall is low.

One major reason for false positives is that fluctuations in the difference value around a dust devil near the camera, which is usually imaged as a large feature, are recognized as different dust devils distinct from the main body of the dust devil. Because these fluctuations in difference value are similar to noise, this kind of false positive may be removed by performing lowpass filtering prior to the binarization. A good example of this problem is seen in detection of dust devils on image No. 9 (Fig. 4). Although true dust devils are included in detected areas, there are small false positives located to the immediate left of the detected area.

There are three reasons for false negatives, which for scientific purposes are more serious than false positives. In cases where a dust devil is small in pixels and optically thin, the dust devil is indistinguishable from noise and is removed as a result. In another type of case, a dust devil has an overlap with another dust devil "ghost" (Fig. 5), which is a dust devil that appears 


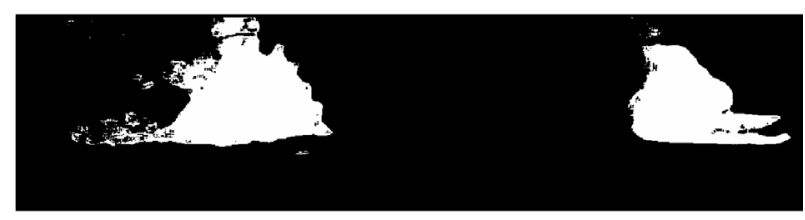

Fig. 4. A binarized image showing many tiny false positives around the main body of a detected dust devil. The input and background images are No. 9 and No. 8, respectively.

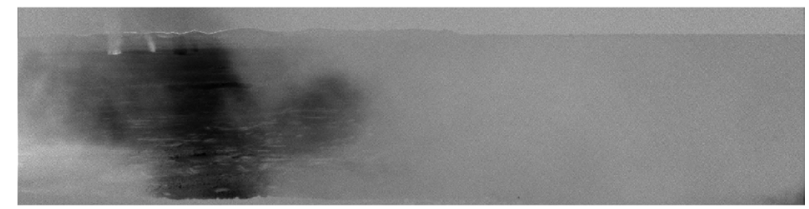

Fig. 5. A difference image showing an example of an overlap between a dust devil and the ghost of another dust devil. The image has been enhanced to emphasize detail. The input and background images are No. 11 and No. 10 , respectively.

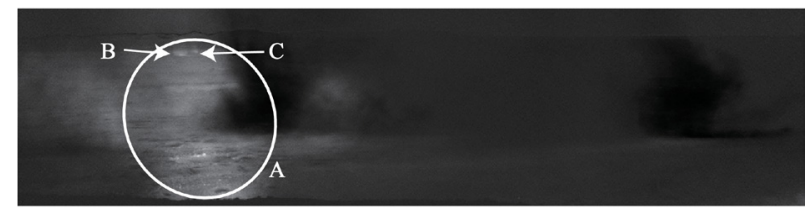

Fig. 6. A difference image showing an example of an overlap between one large dust devil (A) and another two small dust devils (B and C) in a single input image. The image has been enhanced to emphasize detail. The input and background images are No. 10 and No. 9, respectively.

in the background image and is recognized as a dark feature on the difference image. The dust devil to be detected is signicantly darkened in the difference image by the subtraction of the background image from the input image. Therefore, the dust devil cannot be detected as a bright feature in the difference values.

In the third type of case, multiple dust devils overlap each other on a single input image. In the example shown in Fig. 6, two small dust devils (B and C) can not be detected separately from the large dust devil (A). In this case, the two dust devils are included in one detected region and are then recognized as a single dust devil. In other words, the dust devils that are not detected are not a "true" false negatives. If we regard these kinds of false negative as true positives in this study, the number of false negatives is only 3 , and recall $r$ improves to 0.92 .

\section{Conclusion}

A system for automated detection of dust devils in images acquired by a camera aboard a Mars lander or rover has been developed. This system was tested on 21 images taken by the Spirit/Navcam on Sol 0568 (Table 1). Precision, recall, and $F$ values are $0.85,0.81$, and 0.83 , respectively, at worst, comparable to those for the software described by Ref. 7), which are $0.85,0.96$, and 0.90 , respectively. Although the number of images used for this simple test is less than that used by Ref. 7), our precision and recall results are promising because according to the definition of true positive used by Ref. 7), they scored solely based on whether or not the image contained dust devils (i.e., they did not require the locations of the dust devils to be correct). ${ }^{7}$ Furthermore, two dust devils rarely overlap each other in the images. Therefore, the probability of false negatives of the type shown in Fig. 5 is very low; thus, the automated dust devil detection system developed in this study actually has performance higher than that indicated by the values of precision, recall, and $F$.

However, the values of the three indices are lower than those used as standard evaluation criteria in other fields (e.g., machine vision, medical engineering, and pattern recognition). A high rate of false negatives leads to a loss of scientically important information on dust devils; a high rate of false positives leads to an increase in downlinked data volume and time to search for true dust devils among the many false dust devils for meteorological research. We need to further improve both precision and recall in practice.

\section{References}

1) Newman, C. E., Lewis, S. R., Read, P. L. and Forget, F.: Modeling the Martian Dust Cycle 1. Representations of Dust Transport Processes, J. Geophys. Res. E: Planets, 107 (2002), pp.1-18.

2) Ferri, F., Smith, P. H., Lemmon, M., and Renno, N. O.: Dust Devils as Observed by Mars Pathfinder, J. Geophys. Res. E: Planets, 108 (2003), pp.7-1-7-10.

3) Greeley, R., Whelley, P. L., Arvidson, R. E., Cabrol, N. A., Foley, D. J., Franklin, B. J., Geissler, P. G., Golombek, M. P., Kuzmin, R. O., Landis, G. A., Lemmon, M. T., Neakrase, L. D. V., Squyres, S. W., and Thompson, S. D.: Active Dust devils in Gusev Crater, Mars: Observations from the Mars Exploration Rover Spirit, J. Geophys. Res. E: Planets, 111 (2006), pp.1-16.

4) Greeley, R., Waller, D. A., Cabrol, N. A., Landis, G. A., Lemmon, M. T., Neakrase, L. D. V., Hoffer, M. P., Thompson, S. D., and Whelley, P. L.: Gusev Crater, Mars: Observations of Three Dust Devil Seasons, J. Geophys. Res. E:Planets, 115 (2010), Aricle Number E00F02.

5) Moores, J. E., Lemmon, M. T., Kahanpää, H, Rafkin, S. C. R., Francis, R., Pla-Garcia, J., Bean, K., Haberle, R., Newmanh, C., Mischna, M., Vasavada, A. R., de la Torre Juárez, M, Rennó, N., Bell, J., Calef, F., Cantor, B., Mcconnochiem, T. H., Harri, A.-M., Genzer, M., Wong, M. H, Smith, M. D., Martín-Torres, F. J., Zorzano, M.-P., Kemppinen, O., and McCullough, E.: Observational Evidence of a Suppressed Planetary Boundary Layer in Northern Gale Crater, Mars as seen by the Navcam Instrument Onboard the Mars Science Laboratory Rover, Icarus, 249 (2015), pp.129-142.

6) Estlin, T. A., Bornstein, B. J., Gaines, D. M., Anderson, R. C., Thompson, D. R., Burl, M., Castano, R., and Judd, M.: AEGIS Automated Science Targeting for the MER Opportunity Rover, $A C M$ Trans. Intell. Syst. Technol., 3 (2012), pp.1-19.

7) Castano, A., Fukunaga, A., Biesiadecki, J., Neakrase, L., Whelley, P., Greeley, R., Lemmon, M., Castano, R., and Chien, S.: Automatic detection of dust devils and clouds on Mars, Mach. Vis. Appl., 19 (2008), pp.467-482.

8) Maki, J. N., Bell, J. F. III., Herkenhoff, K. E., Squyres, S. W., Kiely, A., Klimesh, M., Schwochert, M., Litwin, T., Willson, R., Johnson, A., Maimone, M., Baumgartner, E., Collins, A., Wadsworth, M., Elliot, S. T., Dingizian, A., Brown, D., Hagerott, E. C., Scherr, L., Deen, R., Alexander, D., and Lorre, J.: Mars Exploration Rover Engineering Cameras, J. Geophys. Res. E: Planets, 108 (2003), pp.ROV 12-1ROV 12-24.

9) Soderblom, J. M., Bell III, J. F., Johnson, J. R., Joseph, J., and Wolff, M. J.: Mars Exploration Rover Navigation Camera in-flight Calibration, J. Geophys. Res. E: Planets, 113 (2008), pp.1-21. 